\title{
Harmonic and Anharmonic Features of IR and NIR Absorption and VCD Spectra of Chiral 4-X-[2.2]Paracyclophanes
}

\author{
Sergio Abbate, ${ }^{*(\mathfrak{E}), \dagger},^{\dagger}$ Ettore Castiglioni, ${ }^{\dagger,},{ }^{\dagger}$ Fabrizio Gangemi, ${ }^{\dagger}$ Roberto Gangemi, ${ }^{\dagger}$ \\ Giovanna Longhi, ${ }^{\dagger}$ Renzo Ruzziconi, ${ }^{*}$, and Sara Spizzichino ${ }^{\S}$ \\ Dipartimento di Scienze Biomediche e Biotecnologie, Università di Brescia, Viale Europa 11, \\ 25123 Brescia, Italy, Dipartimento di Chimica, Università di Perugia, via Elce di Sotto 8, \\ 06100 Perugia, Italy, and JASCO Corporation, Hachioji-shi, Tokyo, Japan
}

Received: March 16, 2007; In Final Form: May 3, 2007

\begin{abstract}
The vibrational absorption spectra and vibrational circular dichroism (VCD) spectra of both enantiomers of 4-X-[2.2]paracyclophanes $\left(\mathrm{X}=\mathrm{COOCD}_{3}, \mathrm{Cl}, \mathrm{I}\right)$ have been recorded for a few regions in the range of 900$12000 \mathrm{~cm}^{-1}$. The analysis of the VCD spectra for the two IR regions, $900-1600 \mathrm{~cm}^{-1}$ and $2800-3200 \mathrm{~cm}^{-1}$, is conducted by comparing with DFT calculations of the corresponding spectra; the latter region reveals common motifs of vibrational modes for the three molecules for aliphatic $\mathrm{CH}$ stretching fundamentals, whereas in the mid-IR region, one is able to identify specific signatures arising from the substituent groups X. In the $\mathrm{CH}$ stretching region between 2900 and $2800 \mathrm{~cm}^{-1}$, we identify and interpret a group of three IR VCD bands due to $\mathrm{HCH}$ bending overtone transitions in Fermi resonance with $\mathrm{CH}$ stretching fundamental transitions. The analysis of the NIR region between $\sim 8000$ and $\sim 9000 \mathrm{~cm}^{-1}$ for $\mathrm{X}=\mathrm{COOCD}_{3}$ reveals important features of the aromatic $\mathrm{CH}$ stretching overtones that are of value since the aromatic $\mathrm{CH}$ stretching fundamentals are almost silent. The intensifying of such overtones is attributed to electrical anharmonicity terms, which are evaluated here by ab initio methods and compared with literature data.
\end{abstract}

\section{Introduction}

Since the first time of their synthesis, ${ }^{1}$ [2.2] paracyclophanes (PC) have been considered interesting molecular systems and have been challenging various physical and chemical techniques in defining diverse aspects tied to their peculiar structure. Several characteristics that are mainly related to the planar chirality of the cyclophane moiety have been investigated, ${ }^{2}$ and recently, there has been a renewed interest to use derivatives of PC in asymmetric catalysis $^{3}$ or to employ them in material science. ${ }^{5}$ Herein, we are merely interested in chiroptical and spectroscopic aspects of some of these still exotic molecules, namely, 4-X[2.2] paracyclophanes $1\left(\mathrm{X}=\mathrm{COOCD}_{3}\right), 2(\mathrm{X}=\mathrm{Cl})$, and $3(\mathrm{X}$ $=\mathrm{I}$ ) (Chart 1 below); yet, we have found results that allow an understanding of the general properties of the moieties of these molecules, namely, the aromatic rings, the polarizable halogen atoms, and the behavior of the methoxycarbonyl group and of bridging aliphatic $\mathrm{CH}_{2}$ groups.

We have been doing so by use of absorption infrared (IR) and near infrared (NIR) spectroscopy, as well as by vibrational circular dichroism (VCD) spectroscopy both in the IR and NIR ranges. We were inspired by precedent research on 4-X-[2.2]paracyclophanes by some of us ${ }^{6,7}$ who were able to relate the observed specific rotation values to the polarizability values of the substituent group ${ }^{6}$ and were also able to interpret the observed circular dichroism spectra in the UV range (CD) in terms of the exciton model ${ }^{8}$ supplemented with due accounting for the influence of the $\mathrm{X}$ group on tilting the transition moments

${ }^{(\mathfrak{f})}$ To whom correspondence should be addressed. Tel.: +39030 3717415. Fax: +39030 3717416. E-mail: abbate@med.unibs.it.

$\dagger$ Università di Brescia.

$\S$ Università di Perugia.

* JASCO Corporation.

\section{CHART 1}

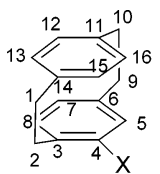

$(R)$

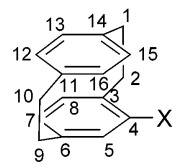

(S)

$$
\mathrm{X}=\mathrm{COOCD}_{3}, \mathrm{Cl}, \mathrm{I}
$$

of the electronic transitions. ${ }^{7}$ Prior to this work, VCD spectroscopy in the $1000-1800 \mathrm{~cm}^{-1}$ range had already been used on some chiral disubstituted [2.2] paracyclophanes, ${ }^{4}$ with the scope of making a safe assignment of the absolute configuration (AC), when charge-transfer effects were verified to obscure the simple interpretation of the UV-CD spectrum provided in ref 7. The scopes of the present work are somewhat different from those of ref 7 since here the AC is known from the beginning; we are going to focus on finer details of the VCD spectra, which will though be of help in understanding the characteristics of the different moieties of the molecules under investigation.

\section{Materials and Methods}

Synthesis and Polarimetric Measurements. The synthesis of both enantiomers of the 4-halogenated [2.2]paracyclophanes, 2 and 3, was reported previously, ${ }^{6}$ and the measurements of the specific optical rotation were described therein. Both enantiomers of 1 were obtained by reacting optically pure $(R)$ and $(S)$-4-carboxy[2.2] paracyclophane ${ }^{6}$ with $\mathrm{SOCl}_{2}$ followed by the DMAP-catalyzed solvolysis of the resulting acyl chloride in $\mathrm{CD}_{3} \mathrm{OH}$. The measurement of the specific rotation for $\mathbf{1}$ has given the following results: $[\alpha]_{\mathrm{D}}{ }^{23}=+148.0\left(c=0.5, \mathrm{CHCl}_{3}\right)$ for $(S)$-4-methoxycarbonyl- $d_{3}-[2.2]$ paracyclophane, and $[\alpha]_{\mathrm{D}}{ }^{23}$ $=-149.7\left(c=0.5, \mathrm{CHCl}_{3}\right)$ for $(R)-4$-methoxycarbonyl- $d_{3-}$ [2.2]- 
paracyclophane. As discussed later, these values give further credit to the interpretation put forward in ref 6 .

IR and IR-VCD Spectra Measurements. IR absorption spectra, IR spectra for short, and IR-VCD spectra were recorded with a JASCO FVS4000 FTIR spectropolarimeter equipped with two detectors, a MCT one and an InSb one. The first one has been used to investigate the mid-IR region, 900-1600 $\mathrm{cm}^{-1}$ and the second one for the $\mathrm{CH}$ stretching region, 2700-3200 $\mathrm{cm}^{-1}$; for the former region, 4000 scans were needed for each spectra $(\sim 40 \mathrm{~min})$, and for the latter 10000 were needed $(\sim 1.2$ h). The spectra of both the enantiomers of $\mathbf{2}$ and $\mathbf{3}$ were recorded for $\mathrm{CCl}_{4}$ solutions (ca. $0.08-0.1 \mathrm{M}$, cell path length $500 \mu \mathrm{m}$ ), whereas the spectra of the enantiomers of 1 were recorded for $\mathrm{CDCl}_{3}$ solutions $(0.5 \mathrm{M}$, cell path length $100 \mu \mathrm{m})$ in $\mathrm{BaF}_{2}$ cells. The IR spectra reported below were obtained by subtracting out the IR spectra of the solvent. The VCD spectra of the enantiomers of each molecule were verified to be mirror images of each other. However, to avoid confusion and for better comparison with calculations, we will report in the following just the average VCD spectra of the two $(R)$ and $(S)$ enantiomers, namely, the VCD data for $(1 / 2)[(R)-(S)]$.

NIR and NIR-VCD Spectra Measurements. NIR absorption spectra, NIR spectra for short, in the range of 1300-800 nm and NIR-VCD spectra in the region of 1250-1050 nm have been recorded with a homemade dispersive apparatus equipped with an InGaAs detector described previously; ${ }^{9,10}$ in the range of 1800-1600 nm, we used a Jasco 470 Plus. Spectra for the first overtone of the $\mathrm{CH}$ stretchings $(\Delta v=2)(1800-1600 \mathrm{~nm})$, for the second overtone of the $\mathrm{CH}$ stretchings $(\Delta v=3)(1250-$ $1050 \mathrm{~nm})$, and for the third overtone of the $\mathrm{CH}$ stretchings $(\Delta v$ $=4)(1000-800 \mathrm{~nm})$ have been recorded for $0.5 \mathrm{M} \mathrm{CDCl}_{3}$ solutions of 1 in $0.5,2$, and $2 \mathrm{~cm}$ quartz cuvettes, respectively. The NIR-VCD spectra in the $\Delta v=3$ region were registered for the same solutions in the same cuvettes as those for the NIR absorption experiments, averaging over four spectra of four scans each. In Figure 5, the spectra of both enantiomers are reported. Cautions in collecting NIR-VCD data for the interested reader may be found in refs 9 and 10 .

Density Functional Theory (DFT) Calculations. As first described by Stephens ${ }^{11}$ and amply used in the VCD literature, ${ }^{12-17}$ the VCD spectra in the IR can be easily assigned when they are accompanied by DFT calculations. For this reason, by use of the GAUSSIAN03 suite of programs ${ }^{17}$ for $\mathbf{1 - 3}(\mathrm{R})$, we ran DFT calculations with B3LYP functionals and with both 6-31G** and TZVP bases; for the iodine atom in compound $\mathbf{3}$, we adopted the $3-21 \mathrm{G}^{* *}$ basis set. By comparing experimental and calculated IR and VCD spectra, we propose that TZVP is a better choice for all three molecular species. Moreover, for $\mathbf{1}$, we have studied two conformers, namely, the one in which the $\mathrm{C}=\mathrm{O}$ in the substituent $\mathrm{X}=\mathrm{COOCD}_{3}$ is closer to (conformer A) or farther from (conformer B) the aliphatic moiety nearby, bridging the two aromatic rings. The two conformers are depicted in Figure 1 and are separated by an $\sim 0.95 \mathrm{kcal} / \mathrm{mol}$ energy difference in the B3LYP/TZVP choice, yielding a population ratio of 84 versus $16 \%$ for $A$ versus $B$ at room temperature (taking into account the Gibbs free energy and the zero point energy, the population of conformer A decreases to $82 \%$ and the one of B increases to $18 \%$ ). Conformation A should then account for almost all of the observed spectroscopic features derived in the present work. For all three molecules, we have not been able to find the two possible skew conformations of the two phenyl rings, as done by Grimme and Bahlmann ${ }^{19}$ for 4-F-[2.2]paracyclophane; we think that, here, bulky substituent $\mathrm{X}$ groups favor the conformation where the nearest methylenic

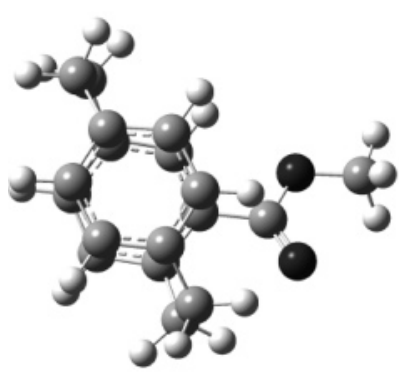

(A)

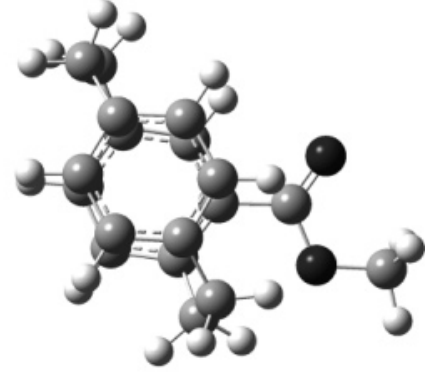

(B)
Figure 1. Calculated conformations of minimum energy for $(R)$-4methoxycarbonyl- $d_{3}-[2.2]$ paracyclophane (1). The conformation on the left is the absolute minimum, and on the right is a secondary minimum (population ratio 84/16 on the basis of B3LYP/TZVP calculations).

hydrogen is away from the phenyl plane. The calculations allow one to come up with harmonic frequencies and dipole and rotational strengths, which, in turn, can be used to generate a calculated IR and VCD spectrum for each molecule by assigning a Lorentzian band shape to each fundamental vibrational transition. We have done so by using a routine in the JASCO FVS4000 software with $\gamma=8 \mathrm{~cm}^{-1}$ ( $\gamma$ is the half-width at halfmaximum) (for the mid-IR region) and $\gamma=16 \mathrm{~cm}^{-1}$ (for the $\mathrm{CH}$ stretching region); in the latter case, a frequency scaling has been adopted in order to facilitate the comparison of calculated and experimental spectra. However, quantum mechanical calculations have been found to be useful also for interpreting some important aspects of the NIR and NIR-VCD spectra. ${ }^{9,17}$ Further and new use has been made here of these calculations, which has allowed us to better understand NIR data.

\section{Results and Discussion}

Each investigated spectral region brings useful information on which we comment below.

The Mid-IR Region (1600-900 $\left.\mathbf{c m}^{-1}\right)$. In Figure 2, the experimental IR (left) and VCD spectra (right) of the three $(R)$ 4-X-[2.2]paracyclophanes $\mathbf{1 - 3}$ (continuous lines) and, superimposed, the corresponding calculated spectra (dotted lines) are reported. As observed in several cases of rigid molecules or in molecules with limited conformational flexibility, ${ }^{11,17}$ the agreement of calculated and experimental IR and VCD spectra is quite good. At first sight, one may grasp interesting differences and analogies among the three molecules. Three groups of bands are worth comment.

i. Region between 1000 and $1100 \mathrm{~cm}^{-1}$. Two intense IR bands with corresponding positive VCD bands are observed and also calculated for 2 and 3 at $\sim 1050$ and $\sim 1010 \mathrm{~cm}^{-1}$, respectively. From the analysis of DFT calculations, we may state that these bands are due to normal mode (NM) 44, involving the following linear combination of $\mathrm{CC}$ stretchings of the ring containing the $\mathrm{X}$ atom: $\left(\mathrm{C}_{6} \mathrm{C}_{5}+\mathrm{C}_{6} \mathrm{C}_{7}-\mathrm{C}_{3} \mathrm{C}_{4}-\mathrm{C}_{3} \mathrm{C}_{8}\right)$, mixed with in-plane $\mathrm{CH}$ bending vibrations of the same ring (see the electronic Supporting Information, ESI). For compound 1, a very intense IR band at $\sim 1100 \mathrm{~cm}^{-1}$, which corresponds to a couple of positive VCD features, is observed. The intense absorption band corresponds to NMs 56 and 57; the first NM is similar to the one described above for compounds $\mathbf{2}$ and $\mathbf{3}$ and bears high rotational strength, the second one involves the $\mathrm{COOCD}_{3}$ moiety, and its frequency is nearly the same in the two conformers. The second VCD band observed at $\sim 1105 \mathrm{~cm}^{-1}$ is due to NM 58 of conformer B (see ESI Tables). 


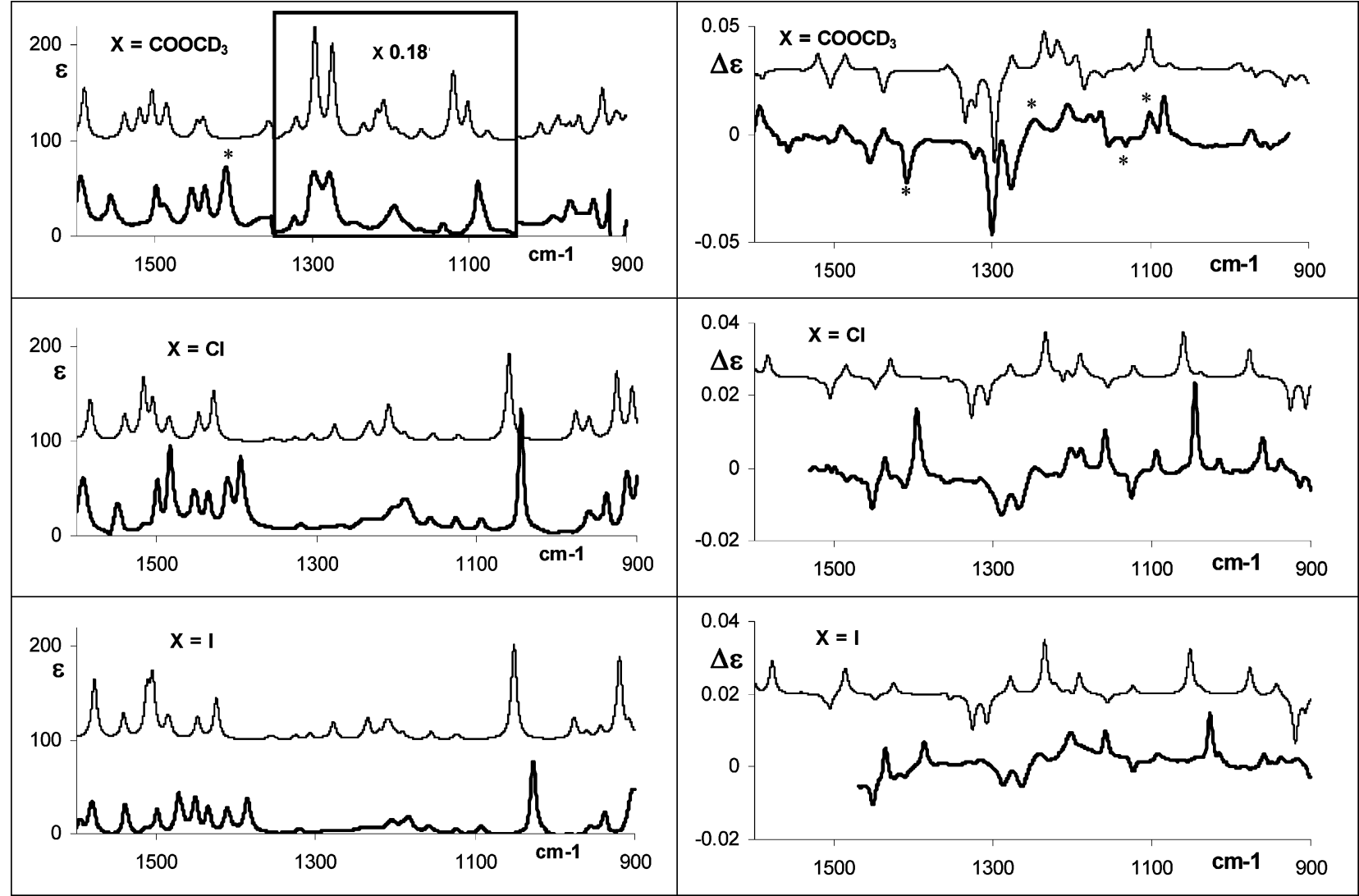

Figure 2. Experimental (bold character) and calculated (light character) IR absorption (left) and VCD (right) spectra in the mid-IR region for $(R)$-4-methoxycarbonyl- $d_{3}$-[2.2]paracyclophane (1) (Top), for $(R)$-4-chloro-[2.2]paracyclophane (2) (Center), and for $(R)$-4-iodo-[2.2]paracyclophane (3) (Bottom). On the experimental IR and VCD spectra of 1, we denote with an asterisk the bands due to the less populated conformer B. In all graphs, $\epsilon$ and $\Delta \epsilon$ are in $10^{3} \mathrm{~cm}^{2} \mathrm{~mol}^{-1}$ units. The inset in the top left panel has the $y$ axis (coinciding with the inset height) reduced by a factor 0.18 so that the full scale is 1100 .

ii. VCD Doublet between 1250 and $1300 \mathrm{~cm}^{-1}$. For the three $\mathrm{X}$-substituted PC, one observes a negative VCD doublet, which is much more intense for $\mathbf{1}$ than for $\mathbf{2}$ and $\mathbf{3}$. In all cases, the DFT calculations allow one to assign these bands to NMs containing important contributions from $\mathrm{CH}_{2}$ wagging and twisting modes of the aliphatic moiety bridging the two aromatic rings on the side of the stereogenic group X. For 1, the $\mathrm{COOCD}_{3}$ group causes the enhancement of the intensity of the doublet both in absorption and $\mathrm{CD}$. This effect can be traced back to the influence of the oxygen atom, as observed in other substituted cyclophanes ${ }^{4}$ and in other cases ${ }^{15,17}$ (see also the comments iv to the data in this section). The negative VCD doublet can be ascribed to the prevalence of conformer A over conformer $\mathrm{B}$; indeed, for conformer $\mathrm{A}$, calculations provide a negative doublet due to the $\mathrm{CO}$ - and $\mathrm{CC}$ stretching vibrations of the two bonds adjacent to the carbonyl, while, for the same NMs, a $(-,+)$ doublet (in order of increasing frequencies) is calculated for conformer B.

iii. VCD Band at $1400 \mathrm{~cm}^{-1}$. This VCD band is in correspondence with a couple of moderately intense IR bands and is negative for $\mathbf{1}$ and positive for $\mathbf{2}$ and $\mathbf{3}$. On the basis of calculated NMs (see ESI material), we assign it to the $\mathrm{CH}_{2}$ modes on the bridging aliphatic moiety opposite to the stereogenic group $\mathrm{X}$ plus in-plane bending modes of nearby $\mathrm{CH}$ bonds on the aromatic ring containing $\mathrm{X}$ (see ESI). Intense negative VCD is associated with conformer B.

$i v$. IR Intensities (Dipole Strengths). The IR spectra of $\mathbf{1}$ in the range of $1050-1350 \mathrm{~cm}^{-1}$ are much more intense than those of $\mathbf{2}$ and $\mathbf{3}$. This is due to the influence of the oxygen atoms, as it was observed previously. ${ }^{4,15,17}$ The calculations presented in 210 Figure 2 reproduce this fact very well.

v. IR and VCD Bands of the Less Populated Conformer. As reported above, conformer $A$ has a population of $\sim 84 \%$ versus conformer $\mathrm{B}$, which is populated at $\sim 16 \%$. This has a nice counterpart in the finding that almost all of the experimental IR and VCD bands are attributed to calculated IR and VCD bands for conformer A (see ESI material, Table and Figure 1). However, as may be seen from the ESI material, there are some isolated and intense VCD and IR bands of conformer B which "survive" the statistical average and show up in the experimental spectra. We have been able to identify four of them in the VCD spectrum at $1407(-), 1246(+), 1131(-)$, and $1100(+) \mathrm{cm}^{-1}$ and one in the IR spectrum at $1437 \mathrm{~cm}^{-1}$; in Figure 2, they are marked with an asterisk, and they suggest a higher population for B than that computed by our in vacuo calculation. In any case, bands associated with the less-populated conformers have been observed in many instances; we cite a very analogous situation, namely, the study of $(3 R)-(+)$-methylcyclohexanone..$^{20}$

As a general comment, we may say that there is an overall similarity in the mid-IR absorption and VCD spectra of $\mathbf{2}$ and $\mathbf{3}$ as opposed to those of $\mathbf{1}$. Only in part can these differences be ascribed to the presence of additional normal modes of the $\mathrm{COOCD}_{3}$ moiety; the $\mathrm{X}$ substituent, in fact, determines also dipole and rotational strengths of other normal modes, for example, in-plane bending of phenyl rings and deformation modes of aliphatic bridges, thus differentiating the spectroscopic behavior of 4-X-[2.2]paracyclophanes. Some analogy may be found with the previous study of ref 6 , where a linear correlation 


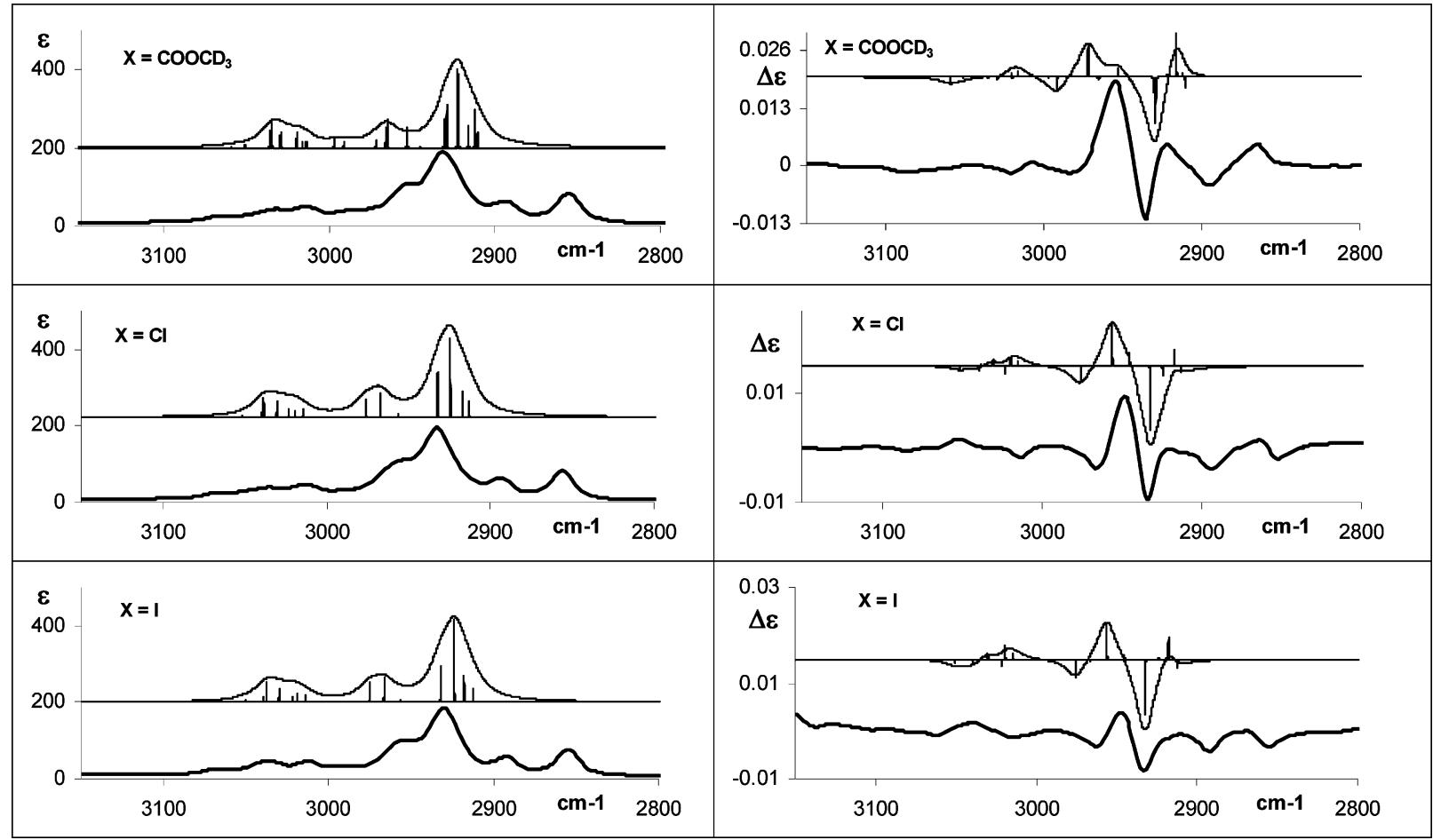

Figure 3. Experimental (bold character) and calculated (light character) IR absorption (left) and VCD (right) spectra in the fundamental CH stretching region for $(R)$-4-methoxycarbonyl- $d_{3}$-[2.2]paracyclophane $(\mathbf{1})$ (Top), for $(R)$-4-chloro-[2.2]paracyclophane $(\mathbf{2})($ Center), and for $(R)$-4iodo-[2.2]paracyclophane (3) (Bottom). Underneath of the calculated IR and VCD bands of $\mathbf{1}$, at the calculated wavenumbers, we report bars that are proportional to calculated dipole and rotational strengths, respectively. In all graphs, $\epsilon$ and $\Delta \epsilon$ are in $10^{3} \mathrm{~cm}^{2} \mathrm{~mol}^{-1} \mathrm{units}_{\text {. }}$

of the specific rotation value $[\alpha]$ with the polarizability value $P_{\mathrm{X}}$ of the $\mathrm{X}$ group was proposed; therein, two distinct correlation straight lines were found between $[\alpha]$ and $P_{\mathrm{X}}$, one for $\mathrm{X}=\mathrm{Cl}$, $\mathrm{Br}$, and I substituents and the other for all other substituent groups X. (Parenthetically, we observe that in ref 6, the case of $\mathrm{X}=\mathrm{COOCD}_{3}$ was not reported in the correlation plot; however, if we consider the relationship $P_{\mathrm{X}}=-0.017[\alpha]+1.58$ being valid, from a mean experimental value of $[\alpha]_{\mathrm{D}}^{23}=-149$, a $P_{\mathrm{X}}$ value of $4.11 \AA^{3}$ can be deduced for the $(R)$ enantiomer. This value is in accord with those $\left(P_{X}=4.17 \AA^{3}\right)$ calculated for the $\mathrm{COOCH}_{3}$ group from the mean polarizability values given in ref 21 for formaldehyde, dimethyl ether, and $\mathrm{CH}_{4}$ and $\mathrm{C}_{2} \mathrm{H}_{6}$, on the basis of a hypothesis of the polarizability additivity.)

Coming back to absorption and VCD spectra, we find much larger dipole and rotational strengths in IR and VCD spectra between 1050 and $1300 \mathrm{~cm}^{-1}$ for $\mathbf{1}$ as opposed to $\mathbf{2}$ and $\mathbf{3}$; also, differences in intensities can be noticed between compounds 2 and 3. Whether this is related to some characteristic invariant of atomic polar tensors, in response to a different substituent group $\mathrm{X}$, remains to be investigated but is beyond the scope of the present study.

The CH Stretching Region (2800-3200 $\left.\mathbf{c m}^{-1}\right)$. The experimental IR and VCD spectra of 1,2, and 3 are quite similar (Figure 3, continuous lines) and comprise three groups of bands, two groups in the aliphatic region (below $3000 \mathrm{~cm}^{-1}$ ) and one group in the aromatic region. The DFT harmonic calculations allow one to get a clue for the assignment of the observed bands between 3200 and $2900 \mathrm{~cm}^{-1}$ as well as to acceptably simulate the observed IR and VCD features in the same range (see Figure 3 , dotted lines). Furthermore, we give here a quantitatively acceptable interpretation of the features in the range of 2800$2900 \mathrm{~cm}^{-1}$ in terms of anharmonic parameters previously derived to interpret the Fermi resonance (FR) phenomenon in $\mathrm{CH}_{2}$ moieties. ${ }^{22,23}$ Let us analyze the spectra in detail.
The Aliphatic Region. a. Bands between 2900 and 3000 $\mathrm{cm}^{-1}$. There is a very intense IR band centered at $\sim 2930 \mathrm{~cm}^{-1}$ with one broad shoulder at $2950-2960 \mathrm{~cm}^{-1}$. In correspondence with it, a couple of evident VCD bands can be observed at $\sim 2930$ and $2950 \mathrm{~cm}^{-1}$ having opposite signs $(-,+)$ for the $(R)$ enantiomer, with rotational strengths that are more intense for $\mathbf{1}$ than for $\mathbf{2}$ and 3. Other VCD features in the same region are observed at $\sim 2920(+)$ and $\sim 2980 \mathrm{~cm}^{-1}$ (-, very weak) for $\mathbf{1}$ and at $\sim 2965 \mathrm{~cm}^{-1}(-)$ for 2 and 3. The calculations allow assignment of the major IR feature at $\sim 2930 \mathrm{~cm}^{-1}$ to the four $\mathrm{CH}_{2}$-symmetric stretching vibrations and the IR shoulder at $\sim 2950 \mathrm{~cm}^{-1}$ to the four $\mathrm{CH}_{2}$-antisymmetric stretchings. Correspondingly, referring to the $(R)$ enantiomer, the pair of major VCD bands with $(-)$ and $(+)$ signs are attributed to superimposed $\mathrm{VCD} \mathrm{CH}_{2}$-symmetric and $\mathrm{CH}_{2}$-antisymmetric vibrational transitions, respectively, bearing mostly (but not exclusively) $(-)$ and (+) signs. The other observed VCD features at frequencies just above and below the major VCD bands are due to lower intensity components bearing different signs embedded in the major VCD features (see Figure 3 and ESI).

b. Bands between 2800 and $2900 \mathrm{~cm}^{-1}$. Looking at the experimental spectra of Figure 3, two IR bands at $\sim 2850$ and $\sim 2890 \mathrm{~cm}^{-1}$, with constant intensity ratio and a triplet of VCD features of alternating sign $(-,+,-)$ in correspondence with them, can be observed for $\mathbf{1}, \mathbf{2}$, and $\mathbf{3}$ (for $\mathbf{1}$, just the + and bands are observed). In the DFT calculations (Figure 3, dotted lines), there is no trace of these features, and we attribute them to strong FR anharmonic phenomena, such that the $\mathrm{CH}_{2}$ bending overtone and combination transitions "acquire" dipole strengths or rotational strengths from nearby $\mathrm{CH}_{2}$-symmetric stretching fundamentals.

To get a first, qualitatively and quantitatively acceptable interpretation of the FR phenomenon, we adopt the approach of ref 22, where the case of $\mathrm{CD}_{3} \mathrm{CH}_{2} \mathrm{CH}_{2} \mathrm{CD}_{3}$ was examined. 
Here, FR is originated within each one of the four $\mathrm{CH}_{2}$ units by an anharmonic interaction between the $\mathrm{HCH}$ bending coordinate $(\delta)$ and the two $\mathrm{CH}$ stretching coordinates $\left(d_{1}\right.$ and $d_{2}$ ). As done in ref 22, we write this interaction Hamiltonian as

$$
\begin{aligned}
V_{\mathrm{anh}}= & f_{\mathrm{d} \delta \delta}\left[\left(d_{1 \mathrm{I}}+d_{2 \mathrm{I}}\right) \delta_{\mathrm{I}}{ }^{2}+\left(d_{1 \mathrm{II}}+d_{2 \mathrm{II}}\right) \delta_{\mathrm{III}}{ }^{2}\right]+ \\
& f_{\mathrm{d} \delta \delta}\left[\left(d_{1 \mathrm{IIII}}+d_{2 \mathrm{IIIII}}\right) \delta_{\mathrm{IIII}}{ }^{2}+\left(d_{1 \mathrm{IV}}+d_{2 \mathrm{IV}}\right) \delta_{\mathrm{IV}}{ }^{2}\right] \\
= & V_{\mathrm{anh}}(1,2)+V_{\mathrm{anh}}(9,10)
\end{aligned}
$$

Here, I, II, III, and IV denote the $\mathrm{CH}_{2}$ units originated from carbon atoms 1, 2, 9, and 10, respectively. As discussed in the Appendix, the anharmonic interaction force constant $f_{\mathrm{d} \delta \delta}$ is an "effective" measure of the anharmonic interaction between bendings and stretchings; in general, one should "divide" it into several contributions, part of them being of kinetic nature, tied to the curvilinear nature of internal coordinates. For now, we settle for the heuristic value derived in ref 22 relative to hexadeuterated $n$-butane. Albeit the merely phenomenological value of this approach, we notice that it was used in the past for several molecules containing $\mathrm{CH}_{2}$ units, and the values of the anharmonic constant $f_{\mathrm{d} \delta \delta}$ were successfully transferred to several molecules containing methylenes. ${ }^{23}$ From the ESI material, we may learn that, as expected, the $\mathrm{CH}$ stretching normal modes involve contributions from either the $(1,2)$ or from the $(9,10)$ moiety but are not mixtures of $(1,2)$ and $(9,10)$ units. As such, we assume, for the $(1,2)$ moiety, a symmetric bending normal mode $Q_{\mathrm{bs}}$ and an antisymmetric bending normal mode $Q_{\text {ba }}$, while there are four stretching normal modes $Q_{\mathrm{sS}}, Q_{\mathrm{aS}}, Q_{\mathrm{sA}}$, and $Q_{\mathrm{aA}}$, which are symmetric or antisymmetric within the $\mathrm{CH}_{2}$ units ( $\mathrm{S}$ or $\mathrm{A}$ ) or between the two $\mathrm{CH}_{2}$ units (s or a). In this simplified scheme, they are tied to the internal coordinates by the relations

$$
\begin{aligned}
& Q_{\mathrm{bs}}=\frac{1}{\sqrt{2}} L_{\delta}^{-1} \delta_{\mathrm{I}}+\frac{1}{\sqrt{2}} L_{\delta}^{-1} \delta_{\mathrm{II}} \\
& Q_{\mathrm{ba}}=\frac{1}{\sqrt{2}} L_{\delta}^{-1} \delta_{\mathrm{I}}-\frac{1}{\sqrt{2}} L_{\delta}^{-1} \delta_{\mathrm{II}}
\end{aligned}
$$

and

$$
\begin{aligned}
& Q_{\mathrm{sS}}=\frac{1}{2} L_{\mathrm{str}}^{-1} d_{1 \mathrm{I}}+\frac{1}{2} L_{\mathrm{str}}^{-1} d_{2 \mathrm{I}}+\frac{1}{2} L_{\mathrm{str}}^{-1} d_{1 \mathrm{II}}+\frac{1}{2} L_{\mathrm{str}}^{-1} d_{2 \mathrm{II}} \\
& Q_{\mathrm{aS}}=\frac{1}{2} L_{\mathrm{str}}^{-1} d_{1 \mathrm{I}}+\frac{1}{2} L_{\mathrm{str}}^{-1} d_{2 \mathrm{I}}-\frac{1}{2} L_{\mathrm{str}}^{-1} d_{1 \mathrm{II}}-\frac{1}{2} L_{\mathrm{str}}^{-1} d_{2 \mathrm{II}} \\
& Q_{\mathrm{sA}}=\frac{1}{2} L_{\mathrm{str}}^{-1} d_{1 \mathrm{I}}-\frac{1}{2} L_{\mathrm{str}}^{-1} d_{2 \mathrm{I}}+\frac{1}{2} L_{\mathrm{str}}^{-1} d_{1 \mathrm{II}}-\frac{1}{2} L_{\mathrm{str}}^{-1} d_{2 \mathrm{II}} \\
& Q_{\mathrm{aA}}=\frac{1}{2} L_{\mathrm{str}}^{-1} d_{1 \mathrm{I}}-\frac{1}{2} L_{\mathrm{str}}^{-1} d_{2 \mathrm{I}}-\frac{1}{2} L_{\mathrm{str}}^{-1} d_{1 \mathrm{II}}+\frac{1}{2} L_{\mathrm{str}}^{-1} d_{2 \mathrm{II}}
\end{aligned}
$$

These assumptions are quite simplifying, but they are not too far from what can be found by the precise normal-mode analysis obtained by use of Gaussian03 (see ESI). The inverse of the above relations may be substituted into eq 1 , obtaining

$$
V_{\mathrm{anh}}(1,2)=f_{\mathrm{d} \delta \delta} L_{\mathrm{str}} L_{\delta}^{2}\left(Q_{\mathrm{ss}} Q_{\mathrm{bs}}^{2}+Q_{\mathrm{ss}} Q_{\mathrm{ba}}^{2}+2 Q_{\mathrm{as}} Q_{\mathrm{bs}} Q_{\mathrm{ba}}\right)
$$

Analogous results can be found for $\mathrm{V}_{\text {anh }}(9,10)$. From eq 2 , for the moiety $(1,2)$ and analogously for moiety $(9,10)$, two interaction matrices, given in the Appendix, can be obtained. The first one is a $(3 \times 3)$ and describes the interaction of the $\mathrm{CH}$ stretching fundamental associated with normal mode $Q_{\mathrm{sS}}$ (anharmonic frequency $\omega_{\mathrm{sS}}-2 \chi_{\mathrm{str}}$ ) and the first overtones of 344 normal modes $Q_{\mathrm{bs}}$ and $Q_{\mathrm{ba}}$ (anharmonic frequencies $2 \omega_{\mathrm{bs}}-6 \chi_{\mathrm{b}} \quad 345$ and $\left.2 \omega_{\mathrm{ba}}-6 \chi_{\mathrm{b}}\right)$; the second one is a $(2 \times 2)$ and describes the 346 interaction of $\mathrm{CH}$ stretching fundamental associated with normal 347 mode $Q_{\mathrm{aS}}$ (anharmonic frequency $\omega_{\mathrm{aS}}-2 \chi_{\mathrm{str}}$ ) with the 348 combination of normal modes $Q_{\mathrm{bs}}$ and $Q_{\mathrm{ba}}$ (anharmonic 349 frequency $\left.\omega_{\mathrm{bs}}+\omega_{\mathrm{ba}}-4 \chi_{\mathrm{b}}\right)$. Here, $\chi_{\mathrm{b}}$ is an effective anharmonic 350 correction, whose value is commented below. The diagonal- 351 ization of these four matrices provides four new frequencies 352 associated with locally symmetric $\mathrm{CH}$ stretchings, plus six 353 frequencies associated with bending overtones and combina- 354 tions; further, it provides normalized eigenvectors, whose first 355 coefficients square may be used to weight the $\mathrm{CH}$ stretching 356 fundamental dipole and rotational strengths, as done in refs $22 \quad 357$ and 23. The matrices are presented in the Appendix. From DFT 358 calculations on molecule 2 , we have obtained for moiety $(1,2) \quad 359$ the values $\omega_{\mathrm{sS}}=3062.2 \mathrm{~cm}^{-1}, \omega_{\mathrm{aS}}=3045.7 \mathrm{~cm}^{-1}, \omega_{\mathrm{bs}}=1504.4360$ $\mathrm{cm}^{-1}$, and $\omega_{\mathrm{ba}}=1485.4 \mathrm{~cm}^{-1}$; for moiety $(9,10)$, the values $\omega_{\mathrm{sS}} 361$ $=3053.8 \mathrm{~cm}^{-1}, \omega_{\mathrm{aS}}=3041.2 \mathrm{~cm}^{-1}, \omega_{\mathrm{bs}}=1504.9 \mathrm{~cm}^{-1}$, and $\omega_{\text {ba }}=1483.3 \mathrm{~cm}^{-1}$ were obtained in the same way (see ESI). As anharmonicity constants, we assumed $\chi_{\text {str }}=60.6 \mathrm{~cm}^{-1}$ and $\chi_{\mathrm{b}}=25$, or 20 , or $15 \mathrm{~cm}^{-1}$, and $f_{\mathrm{d} \delta \delta}=0.201 \mathrm{mdyn} \cdot \mathrm{rad}^{-2}$. We obtained the results of Figure 4 , which we consider quite satisfactory, especially for the case of $\chi_{\mathrm{b}}=15 \mathrm{~cm}^{-1}$ (in Figure 4 , to each transition, we associated a Lorentzian band, centered at the calculated frequency, with the (signed) area equal to the calculated dipole (rotational) strength and with $\gamma=8 \mathrm{~cm}^{-1}$ ). Before commenting on the results, let us discuss how the values for anharmonicity constants were derived. $\chi_{\text {str }}$ has been obtained from experimental IR and NIR CH stretching frequencies fitted to a Birge-Sponer plot $^{24}$ (reported in Figure 3 of ESI, on the basis of the frequencies of experimental NIR absorption bands of Figure 2, ESI). $\chi_{\mathrm{b}}=25 \mathrm{~cm}^{-1}$ was obtained by assuming that the difference between bending harmonic frequencies $\omega_{\mathrm{bs}}$ and $\omega_{\text {ba }}$ calculated with Gaussian03 and of the frequencies for the observed VCD and absorption features at 1443 and 1425 $\mathrm{cm}^{-1}$ (assigned to the fundamental $Q_{\mathrm{ba}}$ and $Q_{\mathrm{bs}}$ normal modes, respectively) was equal to $2 \chi_{\mathrm{b}} \cdot \chi_{\mathrm{b}}=15 \mathrm{~cm}^{-1}$ was instead proposed in ref 26 in a study of overtone spectra of $n$-paraffins, and $\chi_{\mathrm{b}}=20 \mathrm{~cm}^{-1}$ was an intermediate tentative value. Finally, $f_{\mathrm{d} \delta \delta}=0.201 \mathrm{mdyn} \cdot \mathrm{rad}^{-2}$ was derived in ref 22 and used in refs 23 and 26. The aromatic region, not effected by FR, is also reported in Figure 4; it was obtained from the Gaussian03 results by subtracting $\chi_{\text {str }}=65.4 \mathrm{~cm}^{-1}$, as evaluated from the BirgeSponer plot presented in the ESI. In Figure 4, we compare the FR results with those obtained with no FR interaction taking place. In this Figure, we did not adopt an arbitrary frequency shift (see Figure 3); instead, having inserted the contribution of stretching anharmonicity $\chi_{\text {str }}$, we obtained a satisfactory prediction of experimental absorption and VCD spectra with respect to frequencies. On the basis of the complete results of Figure 4 , we propose that the three $(-,+,-)$ VCD features observed below $2900 \mathrm{~cm}^{-1}$ acquired their rotational strength from the $\mathrm{CH}_{2}$-symmetric stretching fundamental; the latter modes gave rise to the intense and unresolved VCD negative feature at $2940 \mathrm{~cm}^{-1}$. The higher frequency region, not involved in FR, is well reproduced by this frequency anharmonic correction treatment. As a general conclusion, we observe that this is an instance where anharmonicity does not obscure the usability of the VCD data; indeed, the VCD spectra are similar for $\mathbf{1}, \mathbf{2}$, and $\mathbf{3}$, and thus, we surmise that the same phenomenon is taking place in the same way for the three molecules. As a final comment and as noticed at the beginning of this paragraph, we remark that the anharmonic interactions of eq 1 may not be . . 


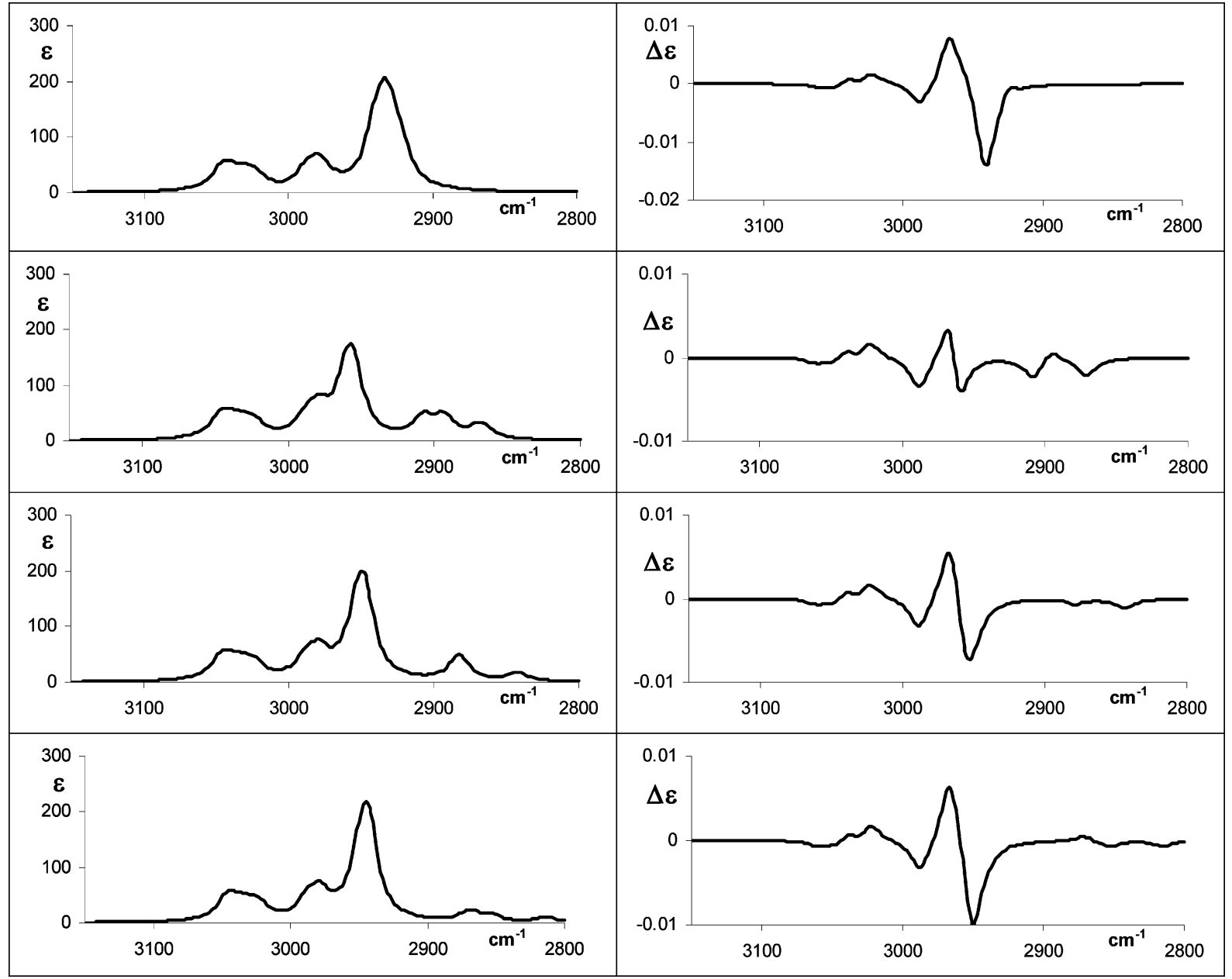

Figure 4. Calculated absorption and CD spectra for molecule 2. From top to bottom: without Fermi resonance (top row), with Fermi Resonance, and $\chi_{\mathrm{b}}=15,20$, and 25 (second, third, and fourth row, respectively) (see text).

a single absorption feature composed of three shoulders. It consists of seven transitions; the lowest frequency features are the three antisymmetric combinations of pairs of $\mathrm{CH}$ stretchings that are localized on each benzene ring on the same side with respect to the aliphatic moiety connecting the two aromatic rings. The highest frequency components of that feature are the corresponding three symmetric combinations and the localized $\mathrm{CH}$ stretching mode of $\mathrm{C}(5)$, which is the closest to the substituent group. The latter vibrational mode has a negative VCD in all three cases and has the highest frequency and does not correlate with experiment, except for $\mathbf{3}$. The other calculated VCD features associated with the other modes can hardly be correlated with the VCD experimental features.

The NIR Region. Considering NIR data are important not only per se but also to obtain parameters useful for improving the interpretation of the IR absorption and IR-VCD spectra. In Figure $2 \mathrm{ESI}$, we show the $\mathrm{CH}$ stretching fundamental absorption spectra for $\mathbf{1}$, for the $\Delta v=1$ region $(3200-3600 \mathrm{~nm})$, the $\mathrm{CH}$ stretching first overtone region $\Delta v=2(1600-1800 \mathrm{~nm})$, the $\mathrm{CH}$ stretching second overtone region $\Delta v=3(1100-1220$ $\mathrm{nm}$ ), and the $\mathrm{CH}$ stretching third overtone region (800-1000 $\mathrm{nm})$. As one may immediately appreciate, the aromatic region is of much weaker intensity than the aliphatic region at $\Delta v=$ 1 but is of comparable intensity at $\Delta v=3$ and 4 . This had been observed previously in other compounds. ${ }^{27,28}$ The rather evident difference between the $\Delta v=1$ and the $\Delta v=2$ and 3 regions is not only due to the local mode regime taking over for $\Delta v \geq 3$ with respect to a normal mode one because of mechanical anharmonicity, but it is also due to high nonlin- 


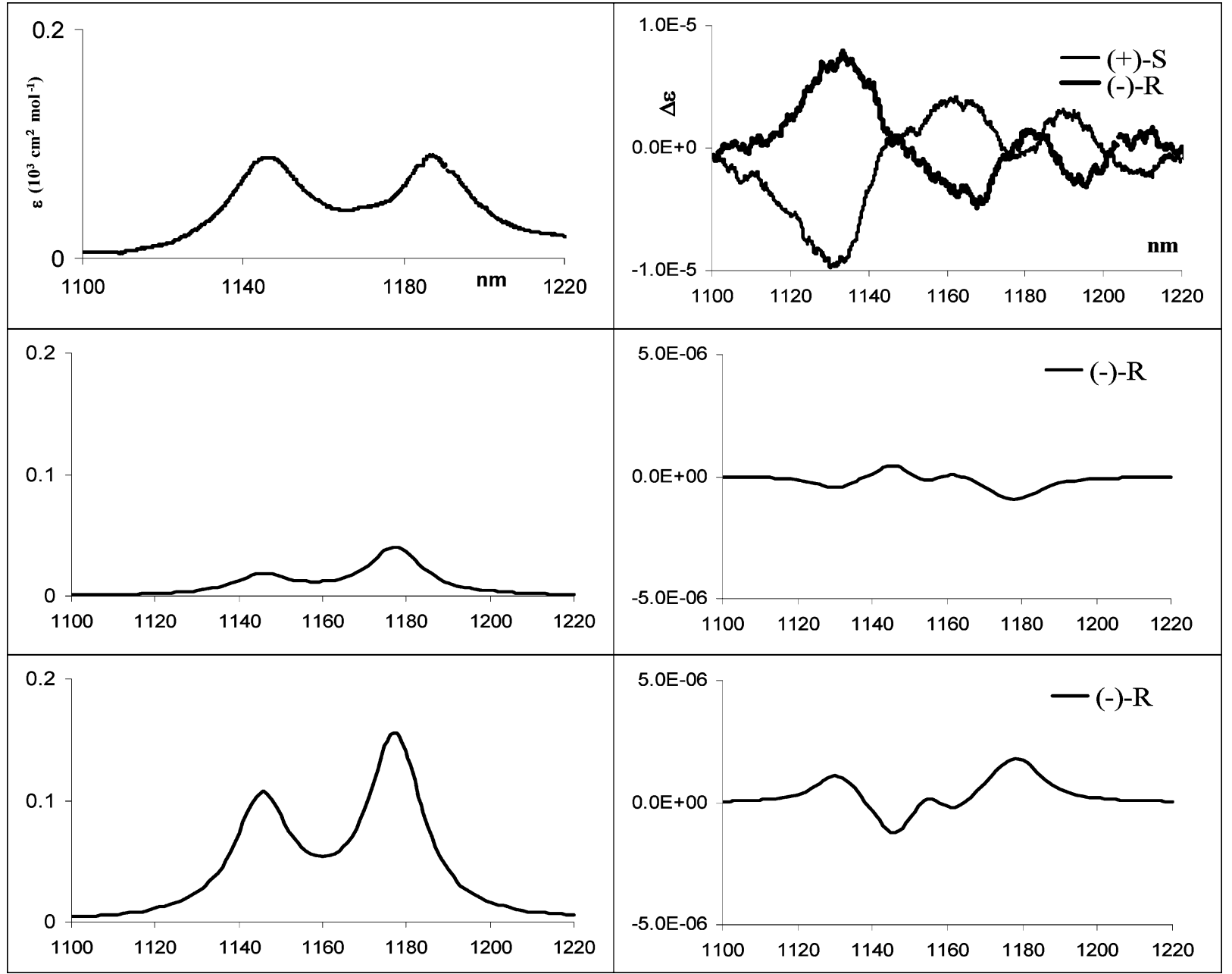

Figure 5. Comparison of experimental (top row, both enantiomers) and calculated ( $R$ configuration) NIR-VCD spectra at $\Delta v=3$ for molecule 1 . Absorption and rotational strengths have been evaluated, as explained in the text in the hypothesis of the electric harmonic approximation (middle row) and with electrical anharmonicity (bottom row).

earities in the $\mathrm{CH}$ bond electrical dipole moment functions. The $\Delta v=2$ region is of intermediate character and thus is hard to decipher. Due to local mode behavior at $\Delta v \geq 3$, one is able to deduce $\omega_{0}$ and $\chi^{24}$ from a Birge-Sponer plot; this is done in Figure $3 \mathrm{ESI}$ and gives $\omega_{0}=3045 \mathrm{~cm}^{-1}$ and $\chi=60.6 \mathrm{~cm}^{-1}$ for aliphatic $\mathrm{CH}$ stretchings and $\omega_{0}=3173 \mathrm{~cm}^{-1}$ and $\chi=65.4$ $\mathrm{cm}^{-1}$ for aromatic $\mathrm{CH}$ stretchings. This is in accord with literature data ${ }^{28}$ and, as we have seen above in Figure 4, allows one to acceptably reproduce the normal-mode observed frequencies at $\Delta v=1$ by subtracting the empirical mechanical anharmonicity $\chi$ from the ab initio-calculated normal-mode frequencies.

While the mechanical anharmonicity can be easily evaluated as done above, the electrical anharmonicity needs to be dealt with in a different way; as it will be seen below, it has important effects on absorption and VCD spectra at $\Delta v=3$. Indeed, in Figure 5, we compare experimental data at $\Delta v=3$ of compound 1 with calculated spectra with and without electrical anharmonicities in the assumption of local modes. In the top two panels of this figure, we report the experimental data (VCD data are for both enantiomers); in the center two panels are the results of a first set of calculations for the $(R)$ enantiomer, performed in the same way as in ref 10 . As done there, we transfer the results of $\mathrm{CH}$ stretching fundamental "local mode" transitions $\Delta v=1$ (where local modes are obtained by considering the molecule with all $\mathrm{H}$ atoms but one substituted by deuterium $)^{10}$ to $\Delta v=3$, applying the hypothesis of electrical harmonicity. We have inserted here the correction factor

$$
\left|\frac{\left\langle 0\left|r-r_{0}\right| v\right\rangle}{\left\langle 0\left|r-r_{0}\right| 1\right\rangle}\right|^{2} \cong \frac{1}{v^{2}} \frac{v !}{(2 K)^{v-1}}
$$

(being $\left.2 K=\omega_{0} / 2 \chi\right)$ for dipole strengths $\left(2.64 \times 10^{-4}\right.$ for 494 aliphatic CHs and $2.83 \times 10^{-4}$ for aromatic CHs, for $\left.v=3\right) 495$ and the correction factor

$$
\frac{\left\langle 0\left|r-r_{0}\right| v\right\rangle\langle v|p| 0\rangle}{\left\langle 0\left|r-r_{0}\right| 1\right\rangle\langle 1|p| 0\rangle} \cong \frac{1}{v} \frac{v !}{(2 K)^{v-1}}
$$

for rotational strengths $\left(7.92 \times 10^{-4}\right.$ for aliphatic $\mathrm{CHs}$ and 8.49 $\times 10^{-4}$ for aromatic $\mathrm{CHs}$, for $v=3$ ). The above factors have been evaluated using Morse wave functions and under the hypothesis that the electric dipole moment function depends linearly on $\left(r-r_{0}\right)$ and the magnetic dipole moment is proportional to $p$; the transition moments for $\left(r-r_{0}\right)$ and $p$ are given in ref 29 and here have been approximated under the hypothesis that $2 K \gg v$. By comparing calculated and experimental results, we see that our model gives dipole and rotational strengths less intense than those observed. As is well-known from the literature, this fact is related to assuming zero electrical anharmonicity. ${ }^{30}$ Furthermore, the aromatic feature at shorter wavelengths is underestimated with respect to the aliphatic one 

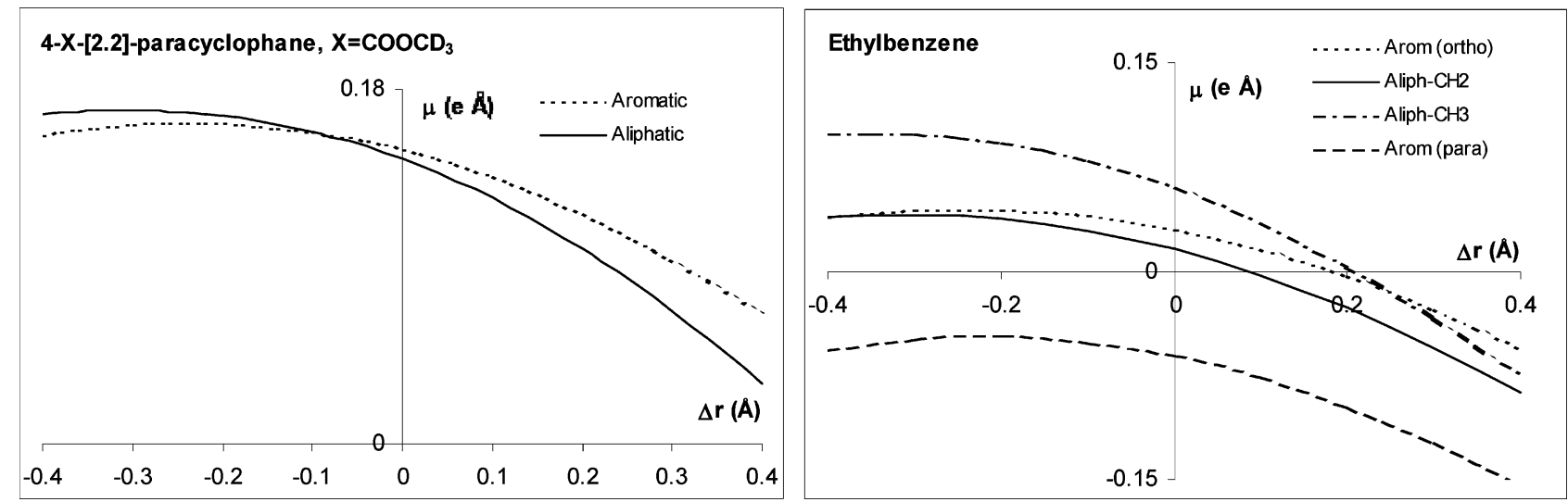

Figure 6. Calculated dependence of the molecular dipole moment function projected onto selected $\mathrm{CH}$ bonds on $\mathrm{H}$-atom displacements along the bond itself for compound 1 (left) and ethylbenzene (right). For the latter molecule, distinction is made between one of the aromatic atoms next to the $\mathrm{CH}_{2} \mathrm{CH}_{3}$ group and the one farthest off; similar calculations have been repeated for an aliphatic methylene $\mathrm{CH}$ and for a methyl $\mathrm{CH}$.

TABLE 1: Comparison of Calculated and Experimental Dipole Moment Derivatives with respect to CH Stretching Coordinates (See Text and Figure 6) ${ }^{a}$

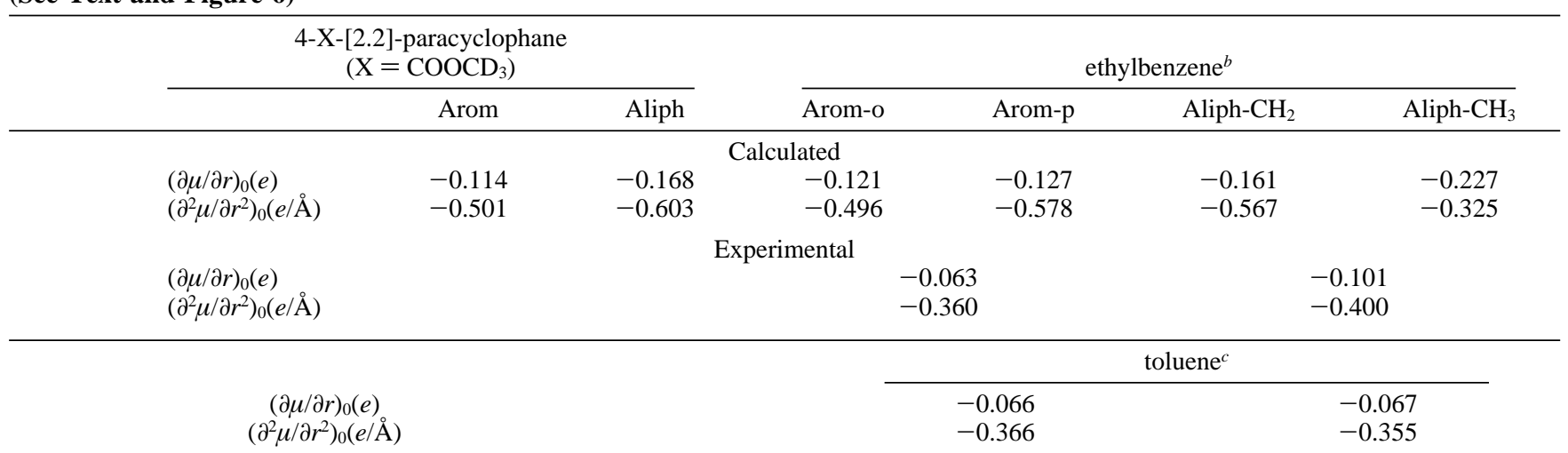

${ }^{a}$ Arom-o: $\mathrm{CH}$ position is ortho to $\mathrm{CH}_{2} \mathrm{CH}_{3}$. Arom-p: $\mathrm{CH}$ position is para to $\mathrm{CH}_{2} \mathrm{CH}_{3} \cdot{ }^{b}$ Experimental values from ref $31 .{ }^{c}$ From ref 27.

both in absorption and in VCD since the calculations follow the prediction of the $\Delta v=1$ region, where they match experiment. Besides, the alternating features of VCD are calculated with an overall $(-1)$ factor, although the alternation of bands seems to be correctly predicted.

In order to cope with the effects of electrical anharmonicity, we have run DFT calculations of electric dipole moment functions, whose results are shown in Figure 6 (B3LYPfunctional, $6-31 \mathrm{G}^{* *}$ basis set). On the left, we provide the calculated components of the electric dipole moment functions along one $\mathrm{CH}$ bond of the aromatic moiety $\left(\mathrm{C}_{12} \mathrm{H}\right.$ in the scheme) and along one $\mathrm{CH}$ bond of the aliphatic moiety $\left(\mathrm{C}_{10} \mathrm{H}\right.$ in the scheme). Such functions have been evaluated by extending and shortening the two $\mathrm{CH}$ bonds in steps of $0.02 \AA$ up to $\pm 0.4 \AA$; the calculated data have been fitted afterward to a fourth-order polynomial, in a similar way to what was done by Henry, Kjaergaard, et al. in ref 31 . We have checked that the fourth-order polynomial coefficients do not significantly vary when the fitting polynomial order is increased up to seven. For sake of comparison, we have also performed calculations for ethylbenzene, and in Figure 6, we report the results for two aromatic $\mathrm{CH}$ bonds (one ortho and one para to the $\mathrm{CH}_{2} \mathrm{CH}_{3}$ group) and for the $\mathrm{CH}$ bonds of $\mathrm{CH}_{2}$ and $\mathrm{CH}_{3}$. As one may immediately see, the first derivatives of such functions, $(\partial \mu / \partial r)_{0}$, are negative and larger in magnitude for aliphatic $\mathrm{CHs}$ than for those for aromatic $\mathrm{CHs}$; this fact justifies the observed feature of higher intensities (dipole and rotational as well) in the aliphatic region at $\Delta v=1$. From the plots in Figure 6, we have derived the values for $(\partial \mu / \partial r)_{0}$ and $\left(\partial^{2} \mu / \partial r^{2}\right)_{0}$ and compare them in Table 1 with experimentally derived values for toluene ${ }^{27}$ and ethylbenzene. ${ }^{32}$ The comparison is rather encouraging. On the basis of eq 6 of refs 27 and 29, we may provide a further correction factor, which includes electrical anharmonicity, to go from $\Delta v=1$ to $\Delta v=v$ local mode intensities

$$
\begin{gathered}
\frac{\langle 0|\mu| v\rangle}{(\partial \mu / \partial r)_{0}\left\langle 0\left|r-r_{0}\right| 1\right\rangle} \cong \\
\frac{1}{v} \sqrt{\frac{v !}{(2 K)^{v-1}}}\left\{1-\frac{1}{a} \frac{\left(\partial^{2} \mu / \partial r^{2}\right)_{0}}{(\partial m / \partial r)_{0}}\left(1+\frac{1}{2}+\ldots+\frac{1}{v-1}\right)\right\}= \\
\frac{1}{v} \sqrt{\frac{v !}{(2 K)^{v-1}}}\left\{1-\frac{1}{a} \frac{\left(\partial^{2} \mu / \partial r^{2}\right)_{0}}{(\partial \mu / \partial r)_{0}} F(v)\right\}= \\
\frac{1}{v} \sqrt{\frac{v !}{(2 K)^{v-1}}} f_{\text {anhar. }}(v)
\end{gathered}
$$

In the latter term, the dimensionless parameter $f_{\text {anhar. }}(v)$ contains the corrections due to electrical anharmonicity. The Morse parameter $a=\left[8 \pi^{2} m c \chi / h\right]^{1 / 2}$ is $1.82 \AA^{-1}$ for aliphatic CHs and $1.89 \AA^{-1}$ for aromatic CHs. In our case, the use of the values for $(\partial \mu / \partial r)_{0}$ and $\left(\partial^{2} \mu / \partial r^{2}\right)_{0}$ calculated for molecule 1 provide, with $f_{\text {anhar. }}(v)=-1.958$, for aliphatic CHs and, with $f_{\text {anhar. }}(v)=$ -2.487 , for aromatic CHs at $v=3$. By applying $f_{\text {anhar }}{ }^{2}(v)$, we obtain calculated absorption spectra that compare quite satis- 
factorily with the observed ones (see the bottom left panel of Figure 5), both with respect to the overall intensities and with respect to the ratio of the aromatic to the aliphatic bands. Interestingly, $f_{\text {anhar. }}(v)$ is negative for both aromatic and aliphatic $\mathrm{CHs}$; this result is due to the fact that $(\partial \mu / \partial r)_{0}$ and $\left(\partial^{2} \mu / \partial r^{2}\right)_{0}$ have the same sign (see Table 1 and Figure 6$)$ and $\mid(1 / a)\left(\partial^{2} \mu \mid\right.$ $\left.\partial r^{2}\right)_{0} F(v)|>|(\partial \mu / \partial r)_{0} \mid$. If we apply the calculated factor to the previous results of Figure 5, center right, we obtain the results for the bottom right panel. The calculated VCD spectrum is of much lower overall intensity than the experimental one, even though signs are in better correspondence with the experiment. We notice that in this treatment of calculated rotational strengths, we have introduced a correction for the electric dipole moment, but we have not taken into account the nonlinearities of the magnetic moment. We do not know yet if this is the only reason for underestimated rotational strengths.

\section{Conclusions}

As previously reported by Furo et al., ${ }^{4}$ this work on $4-\mathrm{X}$ substituted paracyclophanes shows, once more, the possibility to assign the correct $\mathrm{AC}$ by VCD in the mid-IR region combined with ab initio/DFT calculations. The currently commercially available softwares are based on the double harmonic approximation, that is, the assumption of mechanical and electrical harmonicity. However, our study of the IR-VCD spectra in the $\mathrm{CH}$ stretching region has led us to evaluate FR, which determines the $2800-2900 \mathrm{~cm}^{-1}$ portion of the IR and IR-VCD spectra and is due to mechanical anharmonicity. This has been done by transferring an interaction force constant, which has been demonstrated to account well for methylenic signals. The ab initio determination of such an anharmonic force constant is beyond the scope of the present work and is deferred to work like that of Barone et al. ${ }^{26}$ In any case, we may conclude that the use of transferred anharmonic force constants or of ab initio-calculated anharmonic interactions permits one to exploit also the full $\mathrm{CH}$ stretching region to assign $\mathrm{AC}$.

Moreover, in this work, we examined NIR-VCD data, which allowed to shed some light on phenomena related to electrical anharmonicity terms, as pointed out a long time ago on the basis of intensity data of $\mathrm{CH}$ stretching fundamental and overtone transitions. ${ }^{27}$ The procedure proposed here is inevitably approximate due to the complexity of the problem, and in particular, further work needs to be done on the magnetic dipole moment function.

In any case, we think that treating $\mathrm{CH}$ stretchings as isolated Morse oscillators in a chiral field, as proposed some time ago by Polavarapu ${ }^{33}$ and applied in a few studies, ${ }^{10,17}$ is a good starting point for the analysis of NIR-VCD spectra. The correct frame to tackle the problem of electrical and magnetic anharmonicity together with correct handling of mechanical anharmonicity is that proposed by Bak et al. ${ }^{34}$

Acknowledgment. The authors thank MIUR and EULOBrescia for financial support, as participants in a joint project.

\section{Appendix}

Construction of FR Matrices. Due to the form of the anharmonic interaction Hamiltonian, we need to consider the following harmonic wave functions for interacting matrices: $\left|1,0,0,0>=\psi_{1}\left(Q_{\mathrm{ss}}\right) \psi_{0}\left(Q_{\mathrm{as}}\right) \psi_{0}\left(Q_{\mathrm{bs}}\right) \psi_{0}\left(Q_{\mathrm{ba}}\right), \quad\right| 0,0,2,0>=$ $\psi_{0}\left(Q_{\mathrm{ss}}\right) \psi_{0}\left(Q_{\mathrm{as}}\right) \psi_{2}\left(Q_{\mathrm{bs}}\right) \psi_{0}\left(Q_{\mathrm{ba}}\right), \mid 0,0,0,2>=\psi_{0}\left(Q_{\mathrm{ss}}\right) \psi_{0}\left(Q_{\mathrm{as}}\right)$ $\psi_{0}\left(Q_{\mathrm{bs}}\right) \psi_{2}\left(Q_{\mathrm{ba}}\right), \mid 0,1,0,0>=\psi_{0}\left(Q_{\mathrm{ss}}\right) \psi_{1}\left(Q_{\mathrm{as}}\right) \psi_{0}\left(Q_{\mathrm{bs}}\right) \psi_{0}\left(Q_{\mathrm{ba}}\right)$ $\mid 0,0,1,1>=\psi_{0}\left(Q_{\mathrm{ss}}\right) \psi_{0}\left(Q_{\mathrm{as}}\right) \psi_{1}\left(Q_{\mathrm{bs}}\right) \psi_{1}\left(Q_{\mathrm{ba}}\right)$. Two FR interacting matrices are to be considered for moiety $(1,2)$

$\begin{array}{llll} & |1,0,0,0\rangle & |0,0,2,0\rangle & |0,0,0,2\rangle \\ \langle 1,0,0,0| & \omega_{\mathrm{sS}}-2 \chi_{\text {str }} & \Lambda_{1} & \Lambda_{2} \\ \langle 0,0,2,0| & \Lambda_{1} & 2 \omega_{\mathrm{bS}}-6 \chi_{\mathrm{b}} & 0 \\ \langle 0,0,0,2| & \Lambda_{2} & 0 & 2 \omega_{\mathrm{ba}}-6 \chi_{\mathrm{b}}\end{array}$

and

$$
|0,1,0,0\rangle \quad|0,0,1,1\rangle
$$$$
\langle 0,1,0,0| \omega_{\mathrm{aS}}-2 \chi_{\mathrm{str}} \Lambda_{3}
$$$$
\langle 0,0,1,1| \Lambda_{3} \quad \omega_{\mathrm{bs}}+\omega_{\mathrm{ba}}-4 \chi_{\mathrm{b}}
$$

where $\Lambda_{1}=\left(L_{\mathrm{str}} L_{\delta}^{2} f_{\mathrm{d} \delta \delta}\right) /\left(2 H \omega_{\mathrm{sS}}{ }^{1 / 2} \omega_{\mathrm{bs}}\right)=22.96 \mathrm{~cm}^{-1}, \Lambda_{2}=$ $\left(L_{\mathrm{str}} L_{\delta}^{2} f_{\mathrm{d} \delta \delta}\right) /\left(2 H \omega_{\mathrm{sS}}{ }^{1 / 2} \omega_{\mathrm{ba}}\right)=22.32 \mathrm{~cm}^{-1}, \quad \Lambda_{3}=\left(L_{\mathrm{str}}\right.$ $\left.L_{\delta}^{2} f_{\mathrm{d} \delta \delta}\right) /\left(\sqrt{ } 2 H \omega_{\mathrm{aS}}{ }^{1 / 2} \omega_{\mathrm{bs}}{ }^{1 / 2} \omega_{\mathrm{ba}}{ }^{1 / 2}\right)=32.82 \mathrm{~cm}^{-1}$, and $H=$ $\left(8 \pi^{3} c^{5 / 2} 10^{-13} / N_{0}^{3 / 2} h^{1 / 2}\right)$. The frequencies $\omega$, anharmonicities $\chi$, and interaction constants $\Lambda$ are in wavenumbers; $c$ is the light velocity, $N_{0}$ is Avogadro's number, and $h$ is Planck's constant. The constant $H$ given above is such that the anharmonicity constant is mdyne/rad and the eigenvectors' constants $L_{\text {str }}$ and $L_{\delta}$ are in $\mathrm{amu}^{-1 / 2}$ and in $\left(\mathrm{amu}^{-1 / 2} \AA\right)$. We evaluate them from the Wilson $\mathrm{G}$ matrix diagonal elements $L_{\mathrm{str}} \approx G_{\mathrm{rr}}^{1 / 2}$ and $L_{\delta} \approx$ $G_{\phi \phi}{ }^{1 / 2}$ (Wilson, E.B., Jr.; Decius, J.C.; Cross, P.C. Molecular Vibrations; Dover Books: New York, 1980). The matrices above are formally valid for both moieties $(1,2)$ and $(9,10)$; for the latter, small adjustments are needed for slightly different values of $\omega$ 's. By diagonalizing them, one obtains three (two) eigenvalues, which are the frequencies bearing the anharmonic contribution, and for each eigenvalue, normalized eigenvectors with three (two) coefficients, each element of which describes the involvement of each state in the final state. The first coefficient, in all cases, is relative to the fundamental $\mathrm{CH}$ stretching state, and it is the coefficient to consider as responsible for redistributing dipole and rotational strength to anharmonically perturbed bending overtone and combination states. ${ }^{22}$

As pointed out in the main text, the interacting anharmonic Hamiltonian is most probably not complete; following ref 25 , in both moieties $(1,2)$ and $(9,10)$, one should also consider

$$
\begin{aligned}
& V_{\text {anh }}^{\prime}(1,2)=\frac{1}{2}\left(\partial G_{\varphi \varphi} / \partial r_{\mathrm{CH}}\right)_{0}\left[\left(d_{1 \mathrm{I}}+d_{2 \mathrm{I}}\right) p_{\delta \mathrm{I}}^{2}+\right. \\
&\left.\left(d_{1 \mathrm{II}}+d_{2 \mathrm{II}}\right) p_{\delta \mathrm{II}}^{2}\right]
\end{aligned}
$$

where $p_{\delta \mathrm{I}}$ is the momentum conjugate to the coordinate $\delta_{\mathrm{I}}$. One has

$$
\begin{aligned}
&\left(\partial G_{\varphi \varphi} / \partial r_{\mathrm{CH}}\right)_{0}=G^{\prime} \approx-2 r_{\mathrm{CH}}^{-3}\left[1 / m_{\mathrm{H}}+\right. \\
&\left.\left(1-\cos (\mathrm{HCH}) / m_{\mathrm{C}}\right)\right]\left(\mathrm{A} 1^{\prime}\right)
\end{aligned}
$$

From eq A1, following the same methodology in the text, we obtain

$$
\begin{aligned}
V_{\mathrm{anh}}^{\prime}(1,2)=\frac{1}{2}\left(\partial G_{\varphi \varphi} / \partial r_{\mathrm{CH}}\right)_{0} L_{\mathrm{str}} L_{\delta}^{-2}\left(Q_{\mathrm{sS}} P_{\mathrm{bs}}^{2}+\right. \\
\left.Q_{\mathrm{sS}} P_{\mathrm{ba}}^{2}+2 Q_{\mathrm{aS}} P_{\mathrm{bs}} P_{\mathrm{ba}}\right)
\end{aligned}
$$

where $P_{\mathrm{bs}}$ and $P_{\mathrm{ba}}$ are the momenta conjugated to the normal 644 coordinates $Q_{\mathrm{bs}}$ and $Q_{\mathrm{ba}}$. Use of eqs A2 and A1' allows one to 645 evaluate $\Lambda_{1}^{\prime}=-\left(L_{\mathrm{str}} L_{\delta}^{-2} G^{\prime}\right)\left(\omega_{\mathrm{bs}} / \omega_{\mathrm{sS}}{ }^{1 / 2}\right) / 8 H^{\prime}=39.92 \mathrm{~cm}^{-1}, \Lambda_{2}^{\prime} \quad 646$ $=-\left(L_{\mathrm{str}} L_{\delta}^{-2} G^{\prime}\right)\left(\omega_{\mathrm{ba}} / \omega_{\mathrm{sS}}{ }^{1 / 2}\right) / 8 H^{\prime}=39.41 \mathrm{~cm}^{-1}, \Lambda_{3}^{\prime}=-\left(L_{\mathrm{str}} 647\right.$ $\left.L_{\delta}^{-2} G^{\prime}\right) /\left(\omega_{\mathrm{bs}} \omega_{\mathrm{ba}} / \omega_{\mathrm{as}}\right)^{1 / 2} / 4 \sqrt{ } 2 H^{\prime}=56.24 \mathrm{~cm}^{-1}$, and $H^{\prime}=648$ $\left(\pi c^{1 / 2} 10^{-8} / N_{0}{ }^{1 / 2} h^{1 / 2}\right)$. These values are on the same order of 649 magnitude as those for $\Lambda_{1}, \Lambda_{2}$, and $\Lambda_{3}$ that we have used above. 650 These and other kinetic contributions ${ }^{25}$ are always present; 651 651 , .

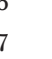

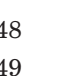
. 
J J. Phys. Chem. A

PAGE EST: 9.7

652

653

654

655

656

657

658

659

660

66

662

663

664

665

666

667

668

669

670

671

672

673

674

675

676

677

678

679

680

681

682

683

684

685

686

687

688

689

691

692

693

694

695

696

697

698

699

700 however, as stated in the text, the ad hoc value $f_{\mathrm{d} \delta \delta}=0.201$ mdyne $\cdot \mathrm{rad}^{-2}$ has guaranteed that the "effective" $\Lambda$ values used in the interacting matrices reproduce the FR scheme well in several molecules containing $\mathrm{CH}_{2}$ groups. ${ }^{22,23}$ For this reason, in the text, we report results ignoring kinetic contribution.

Supporting Information Available: Additional experimental and theoretical results. This material is available free of charge via the Internet at http://pubs.acs.org.

\section{References and Notes}

(1) (a) Brown, C. J.; Farthing, A. C. Nature 1949, 164, 915. (b) Cram, D. J.; Steinberg, H. J. Am. Chem. Soc. 1951, 73, 5691. (c) Vögtle, F. Neumann, P. Synthesis 1973, 85

(2) Vögtle, F. Cyclophane Chemistry, Synthesis, Structure and Reactions; Wiley \& Sons Ltd; Chichester, U.K., 1993; Chapter 2, pp 71-112.

(3) Gibson, S. E.; Knight, J. D. Org. Biomol. Chem. 2003, 1, 1256

(4) (a) Furo, T.; Mori, T.; Wada, T.; Inoue, Y. J. Am. Chem. Soc. 2005 , 127, 8242. (b) Furo, T.; Mori, T.; Origane, Y.; Wada, T.; Izume, H.; Inoue, Y. Chirality 2006, 18, 205

(5) Klee, D.; Weiss, N.; Lahann, J. Vapor-Based Polymerization of Functionalized [2.2]Paracyclophanes: A Unique Approach towards SurfaceEngineered Microenvironments. In Modern Cyclophane Chemistry; Gleiter, R., Hopf, H., Eds.; Wiley-VCH: Weinheim, Germany, 2004; and references cited therein.

(6) Cipiciani, A.; Fringuelli, F.; Mancini, V.; Piermatti, O.; Pizzo, F.; Ruzziconi, R. J. Org. Chem. 1997, 62, 3744.

(7) Rosini, C.; Ruzziconi, R.; Superchi, S.; Fringuelli, F.; Piermatti, O. Tetrahedron: Asymmetry 1998, 9, 55.

(8) Berova, N.; Nakanishi, K. Exciton Chirality Method: Principles and Applications. In Circular Dichroism: Principles and Applications, 2nd ed.; Berova, N., Nakanishi, K., Woody, R. W., Eds.; Wiley-VCH: New York, 2000; Chapter 12, pp 337-382, 7, 161 .

(9) Castiglioni, E.; Lebon, F.; Longhi, G.; Abbate, S. Enantiomer 2002

(10) Longhi, G.; Gangemi, R.; Lebon, F.; Castiglioni, E.; Abbate, S.; Pultz, V. M.; Lightner, D. A. J. Phys. Chem. A 2004, 108, 5338.

(11) (a) Stephens, P. J. J. Phys. Chem. 1985, 89, 748. (b) Stephens, P. J.; Lowe, M. A. Annu. Rev. Phys. Chem. 1985, 36, 213. (c) Stephens, P. J.; Devlin, F. J.; Chabalowski, C. F.; Frisch, M. J. J. Phys. Chem. 1994, 98 , 11623.

(12) Polavarapu, P. L.; He, J. Anal. Chem. A-Pages 2004, 76, 61A.

(13) Nafie, L. A.; Freedman, T. B. Vibrational Optical Activity Theory. In Circular Dichroism: Principles and Applications, 2nd ed.; Berova, N., Nakanishi, K., Woody, R. W., Eds.; Wiley-VCH: New York, 2000; Chapter 4, pp 97-132.

(14) Keiderling, T. A. Peptides and Proteins Confromational Studies with Vibrational Circular Dichroism and Related Spectroscopies. In Circular Dichroism: Principles and Applications, 2nd ed.; Berova, N., Nakanishi, K., Woody, R. W., Eds.; Wiley-VCH: New York, 2000; Chapter 22, pp $621-666$.
(15) Monde, K.; Miura, N.; Hashimoto, T.; Taniguchi, T.; Inoue, T. J. 701 Am. Chem. Soc. 2006, 128, 6000 .

(16) Polyanichko, A.; Wieser, H. Biopolymers 2005, 78, 329.

(17) Longhi, G.; Abbate, S.; Gangemi, R.; Giorgio, E.; Rosini, C. J. Phys. Chem. A 2006, 110, 4958

(18) Frisch, M. J.; Trucks, G. W.; Schlegel, H. B.; Scuseria, G. E.; Robb, M. A.; Cheeseman, J. R.; Montgomery, J. A., Jr.; Vreven, T.; Kudin, K. N.; Burant, J. C.; Millam, J. M.; Iyengar, S. S.; Tomasi, J.; Barone, V.; Mennucci, B.; Cossi, M.; Scalmani, G.; Rega, N.; Petersson, G. A.; Nakatsuji, H.; Hada, M.; Ehara, M.; Toyota, K.; Fukuda, R.; Hasegawa, J.; Ishida, M.; Nakajima, T.; Honda, Y.; Kitao, O.; Nakai, H.; Klene, M.; Li, X.; Knox, J. E.; Hratchian, H. P.; Cross, J. B.; Bakken, V.; Adamo, C.; Jaramillo, J.; Gomperts, R.; Stratmann, R. E.; Yazyev, O.; Austin, A. J.; Cammi, R.; Pomelli, C.; Ochterski, J. W.; Ayala, P. Y.; Morokuma, K.; Voth, G. A.; Salvador, P.; Dannenberg, J. J.; Zakrzewski, V. G.; Dapprich, S.; Daniels, A. D.; Strain, M. C.; Farkas, O.; Malick, D. K.; Rabuck, A. D.; Raghavachari, K.; Foresman, J. B.; Ortiz, J. V.; Cui, Q.; Baboul, A. G.; Clifford, S.; Cioslowski, J.; Stefanov, B. B.; Liu, G.; Liashenko, A.; Piskorz, P.; Komaromi, I.; Martin, R. L.; Fox, D. J.; Keith, T.; Al-Laham, M. A.; Peng, C. Y.; Nanayakkara, A.; Challacombe, M.; Gill, P. M. W.; Johnson, B.; Chen, W.; Wong, M. W.; Gonzalez, C.; Pople, J. A. Gaussian 03, revision B.05; Gaussian, Inc.: Pittsburgh, PA, 2004.

(19) Grimme, S.; Bahlmann, A. Electronic Circular Dichroism of Cyclophanes. In Modern Cyclophane Chemistry; Gleiter, R., Hopf, H., Eds.; Wiley-VCH: Weinheim, Germany, 2004.

(20) Devlin, F. J.; Stephens, P. J. J. Am. Chem. Soc. 1999, 121, 7413.

(21) Ma, B.; Lii, J.; Allinger, N. L. J. Comput. Chem. 2000, 21, 813.

(22) Abbate, S.; Wunder, S. L.; Zerbi, G. J. Phys. Chem. 1984, 88, 593.

(23) (a) Abbate, S.; Zerbi, G.; Wunder, S. L. J. Phys. Chem. 1982, 86, 3140. (b) Ricard, L.; Abbate, S.; Zerbi, G. J. Phys. Chem. 1985, 89, 4793. (c) Longhi, G.; Ricard, L.; Abbate, S.; Zerbi, G. J. Mol. Struct. 1986, 141, 325 .

(24) Herzberg, G. Molecular Spectra and Molecular Structure, I. Spectra of Diatomic Molecules; Van Nostrand Reinhold Co.: New York, 1950.

(25) Ricard-Lespade, L.; Longhi, G.; Abbate, S. Chem. Phys. 1990, 142, 245 .

(26) Barone, V. J. Chem. Phys. 2005, 122, 14108.

(27) Longhi, G.; Zerbi, G.; Ricard, L.; Abbate, S. J. Chem. Phys. 1988, 88,6733 .

(28) (a) Hayward, R. J.; Henry, B. R. Chem. Phys. 1976, 12, 387. (b) Gough, K. M.; Henry, B. R. J. Phys Chem. 1984, 88, 1298.

(29) (a) Sage, M. L. Chem. Phys. 1978, 35, 375. (b) Gallas, J. A. C. Phys. Rev. A 1980, 21, 1829. (c) Longhi, G. Thesis, Università di Milano, Italy, 1984.

(30) (a) Sage, M. L.; Jortner J. Adv. Chem. Phys. 1981, 47, 293. (b) Henry, B. R. Acc. Chem. Res. 1987, 20, 429.

(31) (a) Kjaergard, H. G.; Henry, B. R. J. Chem. Phys. 2004, 96, 4841. (b) see also Rong, Z.; Henry, B. R.; Kjaergard, H. G. J. Phys. Chem. A 2005, 109, 1033 .

(32) Longhi, G.; Abbate, S. 1988, unpublished results.

(33) Polavarapu, P. L. Mol. Phys. 1996, 89, 1503.

(34) Bak, K. L.; Bludsky, O.; Jorgensen, P. J. Chem. Phys. 1998, 103, 10548 . 